\title{
Les mémoires et les correspondances des marins français voyageurs en Angleterre (1764-1785)
}

Memories of French sailors in England (1764-1785)

Sylviane Llinares

\section{OpenEdition}

\section{Journals}

Édition électronique

URL : http://journals.openedition.org/dht/1418

DOI : $10.4000 /$ dht. 1418

ISSN : 1775-4194

Éditeur :

Centre d'histoire des techniques et de l'environnement du Cnam (CDHTE-Cnam), Société des élèves du CDHTE-Cnam

Édition imprimée

Date de publication : 1 décembre 2010

Pagination : $177-185$

ISBN : 978-2-9530779-5-7

ISSN : 0417-8726

Référence électronique

Sylviane Llinares, "Les mémoires et les correspondances des marins français voyageurs en Angleterre (1764-1785)», Documents pour l'histoire des techniques [En ligne], 19 | $2^{\mathrm{e}}$ semestre 2010, mis en ligne le 21 juin 2011, consulté le 08 septembre 2020. URL : http://journals.openedition.org/dht/ 1418 ; DOI : https://doi.org/10.4000/dht.1418 


\title{
Les mémoires et les correspondances des marins français voyageurs en Angleterre (1764-1785)
}

\author{
Sylviane Llinares \\ Université de Bretagne Sud \\ CERHIO - CNRS UMR 6258
}

es marines de guerre sous peine de déclassement _technologique doivent observer, échanger et connaître ce que font leurs rivales. Aux habituelles informations stratégiques sur le déploiement des forces navales d'un État, s'ajoutent celles de nature militaro-industrielle en provenance des ports et des arsenaux. Toutefois, l'activité de renseignement à l'époque moderne emprunte différents chemins et revêt différentes formes dont I'historien s'empare à l'aune des archives conservées. Trois voyages de marins français en Angleterre, dans la seconde moitié du XVIII ${ }^{e}$ siècle, ont heureusement laissé des traces. Henri d'Oraison, François Étienne de Rosily-Mesros et Armand Kersaint, tous les trois officiers, ont obtenu l'autorisation des ministres de la Marine, Étienne-François duc de Choiseul, Antoine Raymond Jean Gabriel de Sartine et Charles-Eugène de la Croix de Castries, de séjourner en Angleterre, de ce fait, chez l'ennemi. Récemment, dans une brillante synthèse sur I'histoire de la marine anglaise, Nicholas Rodger a rappelé les conditions dans lesquelles la Royal Navy avait pu acquérir la maîtrise des mers ${ }^{1}$. Le facteur humain et l'action de l'Amirauté ont été décisifs, permettant de décupler le temps passé à la mer des vaisseaux et des hommes entre la fin du XVII siècle et le milieu du XVIII' siècle. Les méthodes de gestion ont joué pleinement leur rôle dans l'avitaillement des escadres et la mise en œuvre de fournitures navales éprouvées et de qualité, là où il faut, au moment où il faut². En revanche, lorsque l'on étudie les conceptions du navire de guerre en Europe, la mesure de cette formidable avancée est moins évidente à expliquer, bien que le modèle historiographique classique, qui oppose le pragmatisme anglais et la

1 Nicholas A. M. Rodger, The command of the ocean. A naval history of Britain, 1649-1815, Londres, Allen Lane, 2004.

2 Christian Buchet, Marine, économie et société, un exemple d'interaction: I'avitaillement de la Royal Navy durant la guerre de Sept Ans, Paris, H. Champion, 1999. théorisation française, perdure encore. En effet, comme le propose Nicholas Rodger, il faut en premier lieu s'interroger sur l'adéquation des moyens aux besoins. Est-ce que les flottes de guerre de l'époque moderne sont adaptées aux différentes stratégies des États européens ? Ce concept simple permet d'enquêter sur les différents niveaux de réponse aux impératifs de la "politique rationnelle », qu'il s'agisse des systèmes de commandement et d'administration, de l'emplacement des arsenaux ou de la construction navale. Ce que l'on retient est aussi le moment où finalement l'avantage devient manifeste, où les efforts portent leur fruit, où l'optimum d'efficacité se libère, à la charnière chronologique de la guerre de Sept Ans. Ces voyages effectués en 1764, 1776 et 1785 offrent donc un témoignage appréciable sur cette montée en puissance pendant trois décennies qui semblent décisives et à proximité chronologique des deux conflits, de la guerre de Sept Ans et de la guerre d'Amérique. À presque dix ans d'intervalle, ils permettent de confronter le regard de ces officiers sur la marine anglaise à travers une série $d$ 'indices pour lesquels la technique et ses lieux paraissent toujours déterminants. Dans cette approche, le comptage précis des navires et de leurs sabords, exercice factuel un peu fastidieux qui ne nécessite pas obligatoirement I'œil d'un professionnel de la mer, devient secondaire par rapport aux autres types d'informations collectées. Ce que ces marins comparent ou ne comparent pas, ce qui force leur admiration ou fonde leur scepticisme, ce qui retient ou ne retient pas leur attention, méritent une analyse.

\section{Voyages d'agrément et mission d'information}

Les voyages en Angleterre de ces trois marins, qualifiés d'agrément, se doublent d'une mission de renseignement officieuse dont ils doivent rendre compte directement au ministre. Ceux-ci sont libres de leur itinéraire pourvu 
qu'ils mettent à profit leur séjour pour observer la marine anglaise, ses ports et ses navires. Leur personnalité, leur formation et leurs trajectoires professionnelles ont sans doute conditionné leur travail d'observation et les contacts anglais nés de ces voyages ${ }^{3}$.

Ce qui ne s'effectue pas dans le secret, sans les artifices de l'espionnage traditionnel, renvoie à l'idée du voyage et de la production d'information économique et technique dans l'Europe des Lumières que I'on commence à mieux connaître ${ }^{4}$. Les grands ministères d'Ancien Régime ont tous eu de manière plus ou moins organisée des services dédiés au renseignement. Ainsi, les consulats, qui dépendent du secrétaire d'État à la Marine au XVIII' siècle, enregistrent de nombreuses informations sur l'activité maritime d'un État et collectent toutes sortes de données économiques, sociales et politiques ${ }^{5}$. II faut évoquer l'aspect formel des documents produits à cette période. Le « mémoire » est caractéristique des pratiques administratives de la monarchie. Les commis des bureaux de Versailles, les commissaires et intendants des arsenaux, les officiers des vaisseaux du roi, sont tous dans I'obligation de rédiger ce type de document. Le marin qui commande un navire est de toute façon habitué à l'écriture. Un journal de bord est le plus souvent, au final, un gros mémoire qui n'est pas seulement un document technique sur la navigation. Le journal reproduit une information de nature réflexive et événementielle dans une forme très synthétique ${ }^{6}$. Les factums rédigés par les officiers de la marine française voyageant en Angleterre pendant les années 1760-1780 renvoient aussi à la tradition du voyage technique maritime ordonné par Jean-Baptiste Colbert au XVII siècle pour maîtriser les techniques de construction navale européenne ${ }^{7}$. Son fils, Jean-Baptiste Antoine Colbert marquis de Seignelay, qui

3 Sur le rôle des voyages à l'époque moderne : Daniel Roche, Humeurs vagabondes: de la circulation des hommes et de l'utilité des voyages, Paris, Fayard, 2003.

4 Dominique Margairaz et Philippe Minard éd., L'information économique $X V l^{-}-X I X$ siècle, Paris, Comité pour I'histoire économique et financière de la France, 2008.

5 Jörg Ulbert et Gérard Le Bouëdec éd., La fonction consulaire à l'époque moderne, Rennes, Presses universitaires de Rennes, 2006. Le rôle du consul est d'abord celui de la défense des intérêts commerciaux du pays mandataire.

6 Article " Journal de bord », Encyclopédie méthodique Marine, Paris, Panckoucke, 1786, tome 2, p. 561.

7 Sur les missions d'observations et leurs influences dans la construction navale européenne, pour une bibliographie à jour avec les nouveaux apports de la recherche : Jean-Pierre Poussou éd., Les constructions navales dans I'histoire, Revue d'Histoire Maritime, $\mathrm{n}^{\circ} 7$, Paris, Presses de l'université de Paris-Sorbonne, 2007 (notamment les articles très riches de Nicolas A. M. Rodger, Michel Daeffler, José Quintero Gonzáles). lui succède à la tête du secrétariat d'État à la Marine, doit son excellente connaissance du milieu maritime à ces séjours de formation imposés par son père ${ }^{8}$. Durant la première moitié du XVIII siècle, les hommes de l'art, les constructeurs, continuent de visiter les chantiers anglais et hollandais, notamment au moment du renouveau technologique de la flotte de guerre française conduit par Jean-Frédéric Phélypeaux comte de Maurepas ${ }^{9}$. Au cours de son voyage, le constructeur Blaise Ollivier a produit une documentation remarquable sur la construction des navires vers 1730, en Europe. Dans un mémoire de plus de 300 pages, il évalue les pratiques anglaises et hollandaises qu'il compare avec les méthodes en usage en France décrites minutieusement ${ }^{10}$. Après la guerre de Sept Ans, ce sont finalement les officiers qui les remplacent dans la collecte des informations. Ce n'est qu'en 1788, qu'un jeune ingénieur, Alexandre Forfait, effectue à nouveau un voyage en Angleterre, accompagné du commissaire de la Marine Daniel Lescallier ${ }^{11}$. Ce qui ne doit pas conduire

8 En 1669-1670, Etienne Hubac, fils d'un constructeur brestois, voyage en Hollande et en Angleterre (Archives nationales [ensuite AN], Marine B2 fo138), de même Pierre Arnoul, commissaire général du port de Toulon (Bibliothèque nationale de France [ensuite BnF], site Richelieu, Cinq Cents Colbert - 201) et Seignelay (Rochefort, Toulon, Marseille, Italie, Hollande, Angleterre); Éric Rieth "Construction navale à franc-bord au Ponant », Neptunia, n 160, avril 1985, pp. 8-21.

9 En 1729, Julien Geslain, constructeur brestois en poste à Rochefort, séjourne en Angleterre sur ordre du roi. II relève les proportions des navires, des plans et compare les gabarits. Joseph Chapelle, constructeur de Toulon, est en Hollande en 1748. Voir les dossiers personnels des constructeurs dans la série Marine C7 (AN).

10 Sur ordre de Maurepas, Blaise Ollivier séjourne trois mois en Angleterre et six semaines en Hollande en 1737, il rédige son mémoire au cours du voyage. Ses « Remarques sur la Marine des Anglais et des Hollandais faites sur les lieux en l'année 1737 » contiennent aussi des dessins et des schémas, (BnF, Nv. acq., Ms 9482) ; transcription du manuscrit en français et en anglais dans : David H. Roberts, $18^{\text {th }}$ century shipbuilding, remarks on the navies of the English and the Dutch from observations made at their dockyards in 1737 by Blaise Ollivier master shipwright of the king of France, Rotherfield, Jean Boudriot Publications, 1992.

11 AN, Marine B7 $475 n^{\circ} 24$ "Mémoire de MM. Forfait et Lescallier par lequel ils rendent compte à $\mathrm{M}$. de La Luzerne de leurs observations sur la Marine pendant leur séjour en Angleterre " 1788. Voir Margaret Bradley, Daniel Lescallier, 1743-1822, man of the sea and military spy?: maritime developments and French military espionage, Lewiston, New York Edwin Mellen Press, cop. 2005, et Sylviane Llinares, Marine, propulsion et technique au XVIIle siècle, 2 vol., Paris, Librairie de I'Inde, 1994. En 1775, Lescallier visite les ports anglais, russes et suédois, il publie en 1800 la relation de ce séjour : Voyage en Angleterre, en Russie et en Suède, fait en 1775, Paris, Firmin 
à réfuter l'idée de la circulation des ingénieurs dans un espace technique européen au siècle des Lumières. Certains ingénieurs ont pu avoir des informations indirectes, tel Jean-François Gautier qui œuvre dans les arsenaux espagnols (1769-1784), mais il n'en reste pas moins vrai que les très grands ingénieurs de la Marine, Antoine Groignard ou Jacques Sané, ne quittent guère les ports de France. Les voyages en Angleterre de ces trois marins, d'Oraison, Rosily et Kersaint sont donc inédits, d'où leur réel intérêt ${ }^{12}$.

Qui sont-ils ? Leurs noms sont peu connus hormis chez les spécialistes de I'histoire maritime du XVIII' siècle. Nul doute cependant que l'évocation de Louis-Antoine Bougainville, de Yves Joseph de Kerguelen ou de la Belle-Poule rende plus lisible la vocation maritime de ces hommes et aiguise un peu la curiosité biographique ${ }^{13}$. Henri d'Oraison est né à Aix-en-Provence en janvier 1739, il est garde de la Marine à Toulon en mai 1757 et devient enseigne de vaisseau en novembre 1765. C'est après son séjour en Angleterre qu'il embarque sur la frégate la Boudeuse commandée par Bougainville pour un tour du monde, à la fin de l'année 1766. De retour en mars 1769 , il est « retiré du service » pour raison de santé. ॥ touche une pension de 400 livres sur les Invalides de la Marine et décède en 1772, à l'âge de 33 ans, avec le grade de lieutenant de vaisseau ${ }^{14}$. Trois documents, une lettre et deux mémoires, soit une cinquantaine de pages sont conservés dans la série Marine B7 des Archives nationales (Pays étrangers, commerce et consulats): un «Mémoire sur l'Amirauté anglaise contenant la description de Portsmouth et Plymouth » daté de 1764, un «Mémoire sur les arsenaux et chantiers anglais, Chatham, Sheerness, Deptford, Blackwall, Woolwich » daté de 1765 et une lettre de La Haye datée du 17 janvier 1765 portant mention de l'expédition du mémoire au ministre, Choiseul ${ }^{15}$ :

«Monseigneur, Je viens de remettre à Monsieur le Comte de G... un mémoire qui contient les descriptions de Chatham, Sheerness, Woolwich, Deptford et Blackwall avec les proportions de construction

Didot, an VIII, $174 \mathrm{p}$.

12 Martine Acerra et José Merino, «Jean-François Gautier, vie et influence d'un ingénieur marginal », Neptunia, n 158, juin 1985, pp. 6-12.

13 Voir les notices dans Étienne Taillemite, Dictionnaire des marins français, Paris, Taillandier, 2002 (Rosily-Mesros, p. 464 et Kersaint, p. 275 dont les séjours en Angleterre sont signalés ; pas de notice en revanche sur Henri d'Oraison).

14 AN, Marine C7 231 : dossier Personnel « d'Oraison ».

15 AN, Marine B7 475: $n^{\circ} 11$ et 12 . Cette série s'intitule « Mémoires et documents concernantles marines étrangères 13891793 », B7 473 à 475 : Angleterre (1389-1793). mâture et cordages des vaisseaux anglais, ces objets avec les observations que j'ai déjà eu l'honneur de vous envoyer par la voie de Monsieur l'ambassadeur, complètent relativement à la marine tout ce qui mérite de vous être envoyé de ce pays cy ${ }^{16}$.

François Étienne de Rosily-Mesros est né à Brest en janvier 1748. II est enseigne de vaisseau en 1770 et a déjà fait plusieurs campagnes dans l'Atlantique avant son séjour en Angleterre. II a notamment voyagé avec Kerguelen jusqu'aux Terres Australes, en 1771-1772. Scorbutique en 1774, il débarque à Lorient au bout de 14 mois de campagne sur la corvette l'Ambition, de retour de I'océan Indien. Au début de la guerre d'Indépendance américaine, il commande le lougre le Coureur et s'illustre dans le célèbre combat de la Belle-Poule du 17 juin 1778, où il est fait prisonnier et le reste jusqu'en février 1780. II participe ensuite à la campagne des Indes avec Suffren en 1782-1784, un espace maritime qu'il ne quitte plus jusqu'en 1790. II effectue une formidable campagne sur la Vénus de 1784 à 1788, jusqu'en Chine et croise ensuite aux Philippines et en Cochinchine sur la Méduse. Son expérience dans le domaine hydrographique lui vaut d'être nommé directeur général du Dépôt des cartes et plans de la Marine en 1795. II poursuit une brillante carrière sous I'Empire et meurt à Paris en 1832. La documentation sur son voyage en Angleterre, une mission approuvée par le ministre Sartine, est dispersée et peu identifiable de prime abord. On retrouve un petit mémoire de douze pages "Chantiers d'Angleterre pour M. de Sartine » dans la série Marine B7 (anonyme) et un autre mémoire de 25 pages sur «I'Administration de la Marine anglaise, Mémoire du Comte de Rosily du 27 décembre 1776 au 21 mars 1777 » dans la série Marine G. Son dossier personnel contient des extraits de plusieurs lettres que le ministre Sartine lui a envoyées en Angleterre de septembre 1776 à janvier 1777 ${ }^{17}$. Des éléments de la documentation collectée par Rosily sur les pompes à chaînes anglaises constituent une partie des pièces d'un dossier nommé «Pompes et poulies inventées par les Sieurs Cole et Taylor 1768-1778» provenant de la série des Mémoires et Projets de la Marine ${ }^{18}$. En

16 lbid.

17 AN, Marine B7 $475 n^{\circ}$ 16, Marine G $149 n^{\circ}$ 38. SHD Vincennes, Marine CC7, dossier personnel "Rosily », les lettres expédiées de Versailles sont datées du 13 septembre, 22, 14 et 25 décembre 1776 et du 11 janvier 1777.

18 AN, Marine G 108, dossier 1, $f^{\circ} 20$ : lettre de Londres du 5 décembre 1775, f॰39 et f॰44 : lettres de Londres du 18 mai 1775. Voir l'inventaire analytique, Inventions techniques dans la Marine 1641-1817. Mémoires et projets reçus par 
recoupant les dates et les écritures, on retrouve dans ce dossier les pièces qui étaient jointes aux lettres de Rosily ${ }^{19}$.

Armand Kersaint, né à Paris en 1742, est connu pour être un des rares marins français guillotinés pendant la Terreur, le 24 décembre 1793. Ce qui fait de lui, selon ses biographes, au pire, un traître n'ayant pas émigré, au mieux, une victime des Montagnards qui n'a pas voté la mort du roi. Sa fille, la duchesse de Duras, est connue pour son attachement à Chateaubriand. Le père de Kersaint, qui commandait le Thésée, disparaît dans le terrible combat des Cardinaux du 21 novembre 1759. En 1785, Kersaint, qui est un peu plus âgé que ses prédécesseurs lorsqu'il part I'Angleterre (il a 43 ans), est surtout célèbre pour avoir repris aux Anglais pendant la guerre d'Indépendance américaine, en janvier 1782, les colonies de Guyane hollandaise, Demerara, Essequibo et Berbice. Précisons qu'il fut embarqué à treize ans, comme pilotin avec son père et qu'il a passé presque une vingtaine d'années de sa vie en mer (1755-1787) ${ }^{20}$. Les conséquences du séjour de Kersaint en Angleterre sont importantes en raison de l'armement d'un vaisseau expérimental qu'il obtient du ministre Castries en 1787. Tout ce qu'il a pu comparer et observer en 1785 est mis à profit pour perfectionner le vaisseau de 74 canons, fleuron de la flotte de guerre française ${ }^{21}$. Signalons aussi que ses papiers personnels offrent une correspondance copieuse qu'il faudrait lire attentivement et des documents autobiographiques qui éclairent sur ses contacts en Angleterre. L'on apprend ainsi que c'est « son ami » le banquier Le Couteulx qui I'aide et le recommande à « Monsieur Gloser de Birmingham » afin que celui-ci lui fasse visiter les manufactures dont certaines produisent pour la Royal Navy des toiles, des poulies, etc. Précisons que les Le Couteulx liés au négoce rouennais sont, tout comme Kersaint, actionnaires des

le Département de la Marine (Marine G 86 à G 119), Paris, Archives nationales, 1990, pp. 238-241 et Sylviane Llinares « Les archives de l'invention de la marine de guerre, réflexion pour une exploitation de la série G (XVIIle-XIXe siècles) », dans MarieSophie Corcy, Christiane Demeulenaere-Douyère, Liliane HilairePérez éd., Les archives de l'invention : écrits, objets et images de l'activité inventive, des origines à nos jours, Toulouse, CNRS/ université de Toulouse-Le Mirail, coll. "Méridiennes», série « Histoire et techniques », 2006, pp. 363-377.

19 Spécialement le plan de la pompe à chaîne inventée par William Cole. Voir en annexe le dessin. AN, Marine G 08 Dossier $1 f^{\circ} 6$ " Description of the improved chain pump by John Bentinck Esq., captain in His Majesty's Royal Navy, and William Cole, engine maker », un folio imprimé, s. d.

20231 mois et 26 jours. AN, Marine C7 153 : dossier Personnel de Armand-Guy-Simon de Coëtnemprun, comte de Kersaint.

$21 \mathrm{~S}$. Llinares, Marine, propulsion et technique au XVIII siècle, op. cit. note 11 . nouvelles fonderies de Romilly-sur-Andelle établies pour fabriquer les plaques de cuivre laminé destinées au doublage des carènes des vaisseaux de guerre ${ }^{22}$. Ajoutons pour finir que Kersaint, « Girondin », a porté les revendications du parti « industrialiste » dans le débat sur les orientations du commerce extérieur des années 17901792 et qu'il a plaidé pour l'interdiction des importations de textiles indiens en France. Sa personnalité engage I'historien à une certaine prudence, d'autant que ses deux frères cadets, marins aussi, ont connu des trajectoires différentes et qu'ils sont parfois confondus ${ }^{23}$.

\section{Observation et comparaison}

Le tableau ci-dessous présente les lieux visités dans l'ordre qui figure sur les mémoires. Les trois grandes aires navales (Portsmouth, Tamise et Medway) ont été vues et bien étudiées. À quelques exceptions près, les circuits de visite des ports anglais sont identiques, certains étant incontournables, tels Portsmouth, Woolwich ou Chatham.

Itinéraire des principaux sites portuaires visités

\begin{tabular}{|l|l|l|l|}
\hline & $\begin{array}{l}1764 \\
\text { d'Oraison }\end{array}$ & $\begin{array}{l}1776 \\
\text { Rosily }\end{array}$ & $\begin{array}{l}1785 \\
\text { Kersaint }\end{array}$ \\
\hline Blackwall & 6 & - & 4 \\
\hline Chatham & 3 & 1 & 6 \\
\hline Deptford & 5 & 3 & 3 \\
\hline Plymouth & 2 & - & - \\
\hline Portsmouth & 1 & 2 & 2 \\
\hline Sheerness & 4 & - & 7 \\
\hline Woolwich & 7 & 4 & 5 \\
\hline Bristol & - & - & 1 \\
\hline
\end{tabular}

Par rapport aux voyages précédents, celui du comte de Kersaint présente une nouvelle région d'investigation avec la côte ouest. Bristol qui reçoit tous les bois du pays de Galles est, selon Kersaint, un site très favorable que l'on doit considérer « comme le port le plus intéressant après Londres $»^{24}$. Voici son itinéraire détaillé qui renseigne sur la manière dont il a organisé son séjour en Angleterre :

22 Les papiers du comte de Kersaint (Armand-Guy) sont aux Archives départementales des Yvelines (Versailles), titre de famille E 1426 à 1453.

23 Joseph Guy Marie (1746-1797), chevalier de Kersaint qui passe en Inde et décède à I'lle de France, Guy-Pierre (1747-1822) qui émigre en 1790 , est réintégré en 1798 , chef militaire du port d'Anvers en 1806, chantier du canal de l'Escault en 1810, préfet de la Meurthe en 1815.

24 AN, Marine B7 $475 n^{\circ} 20$, «Vues générales sur l'état actuel de la marine d'Angleterre », août 1785 . 
«Partie ouest: Sur le cours de la Severn, Gloucester et Newham, Cheptown sur la rivière du Wye, Newport harbourg sur la rivière d'Usk, navigation sur le canal Saint-Georges depuis Newport jusqu'à Bristol et sur la rivière d'Avon, examen attentif de la rade de Bristol. Partie sud : Southampton harbourg, Limington crike, navigation dans le canal qui sépare l'île de Whight, $1^{\circ}$ jusqu'au château d'Hurst et Weymouth harbourg, $2^{\circ}$ de Weymouth à Cowes, $3^{\circ}$ de Cowes à Newport par la rivière, par terre de Newport à Ride-Key, $4^{\circ}$ de Ride-Kay en rade de Spithead, Portsmouth et Gosport, $5^{\circ}$ en navigation par le port de Portsmouth jusqu'à Fareham et retour à Portsmouth. Partie est: Navigation sur la Tamise du pont de Londres à Deptford, à Greenwich, à Blackwall, à Woolwich, $2^{\circ}$ voyage à Gravesend et Tilbury clefs de la Tamise, $3^{\circ}$ voyage à Chatham, navigation sur la Medway, dans le port de Chatham, $4^{\circ}$ voyage à Sheerness forteresse arsenal et clef du Medway, $5^{\circ}$ voyage à Margate à la pointe méridionale de l'embouchure de la Tamise, $6^{\circ}$ à Ramsgate port tout neuf sur la rade des dunes, $7^{\circ}$ à Sandwich, $8^{\circ}$ à Deal ville des pilotes pour la Tamise, $9^{\circ}$ de Deal à Douvres ${ }^{25}$.

Les conditions dans lesquelles s'effectuent ces visites sont difficiles à préciser, mais les officiers français ont pu, semble-t-il, accéder aux chantiers et aux magasins sans trop d'obstacles. Henri d'Oraison a pu y pénétrer bien que la surveillance ait été renforcée depuis peu dans les trois grands ports de Portsmouth, Plymouth et Chatham, gardés par des militaires » dont on peut tromper «leur exactitude » précise-t-il. Malgré les interdictions faites aux étrangers de visiter les arsenaux, Rosily " trompe » lui aussi facilement la surveillance à Portsmouth où «le chantier est aussi mal gardé que le port ». II explique que " quand un homme avec un peu de front s'est mis dans la tête d'y entrer, il le peut » et dit être entré « en se faisant mettre à terre sur le rivage du côté des chantiers » et en avoir fait le tour « avec la connaissance de tout ce qui I'a frappé, ayant déjà vu un chantier dans le plus grand détail ${ }^{26}$. C'est on ne peut plus clair ! D'ailleurs Rosily explique dans son mémoire "qu'il y a si peu d'ordre dans la manière dont se fait le service en Angleterre que toutes les personnes qui parlent anglais, peuvent sans aucun risque prendre des gens de mer pour bavarder sur ce qu'ils savent, en deux ou trois promenades sur mer un homme est en état de juger de tout aussi nettement

25 Ibid., « Itinéraire du voyage du comte de Kersaint relativement à la Marine d'Angleterre ».

26 AN, Marine B7 $474 n^{\circ}$ 16, Mémoire anonyme pour Monsieur de Sartine "Chantiers d'Angleterre », que nous attribuons au comte de Rosily.

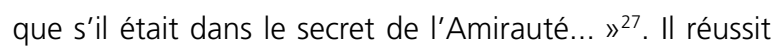
même à visiter un vaisseau de 64 canons à Woolwich, visite essentielle qui déclenche la quête du mécanicien inventeur des pompes de cale observées à bord qu'il juge particulièrement efficaces.

Les observations contenues dans les différents mémoires concernent les aménagements portuaires (bassin de radoub, cales), les équipements spécifiques (forges, fonderies, corderies, ateliers, magasins, machines diverses, etc.), I'organisation des chantiers et du travail, les approvisionnements et la qualité des matières premières, et tout ce qui touche au perfectionnement du navire, par exemple le doublage des coques, I'adoption de nouveaux canons, de poulies, etc. S'y ajoutent l'évaluation du nombre de navires de guerre et de leur puissance de $\mathrm{feu}^{28}$. Rosily a comptabilisé les vaisseaux et frégates de la flotte anglaise, les bâtiments en construction, en radoub, au mouillage et en commission ${ }^{29}$. La correspondance de Sartine avec Rosily met en avant tout ce qui a trait au gréement dans les procédés de fabrication, le ministre lui demandant de ne rien négliger sur les voiles, les cordages, les poulies dans sa mission d'information. Certains aspects ont retenu davantage son attention. II ressort de la lecture du mémoire qu'il a été frappé par le volume des stocks de bois disponibles et par la grève (achevée) des ouvriers. L'humain revient au premier plan, enfin, car les questions de main-d'œuvre sont tout aussi essentielles pour armer une flotte de guerre. II tente de chiffrer le nombre d'ouvriers dans chaque port, ainsi à Portsmouth, où ils ne sont pas plus de 300, y compris les apprentis et les enfants : « il n'y en pas plus à la charpente et à la construction, c'est cette classe qui s'est révoltée et qui a parlé le plus haut » ${ }^{30}$. À Woolwich, il essaie d'analyser la grève bien structurée des charpentiers :

"La révolte qu'il y a eu parmi les charpentiers à été la plus violente à Woolwich que partout ailleurs, il y a eu une circonstance fort singulière dans cette révolte, c'est la nomination d'un correspondant dans chaque chantier, chargé de traiter avec les autres à l'unanimité de toutes les volontés; tous les ouvriers de chaque chantier ayant proposé les mêmes choses et fait les mêmes difficultés. La plus profonde misère a désuni enfin ces malheureux qui manquant de pain ont été

\section{Ibid.}

28 Le chevalier d'Oraison compte 144 vaisseaux et 149 frégates ou corvettes, la flotte française totalise 64 vaisseaux et 50 frégates ou corvettes au départ de Choiseul du ministère de la marine, en 1766.

29160 dans les ports et sur les chantiers, 50 en commissions ou déjà en mer.

$30 \mathrm{lbid}$. 


\section{Les mémoires et les correspondances des marins français voyageurs en Angleterre (1764-1785)}

obligés de se débander ou de prendre de l'ouvrage ; quelques uns se sont rendus en Amérique. Jamais la France n'aura une si belle occasion d'avoir des ouvriers qu'elle l'eut eue dans cet instant de division $»^{31}$.

Cette question des coûts de construction et de main d'œuvre reste présente dans les observations de Kersaint qui a analysé la construction des vaisseaux de guerre sur les chantiers privés. II explique les avantages du système anglais qu'il estime plus économique puisque la formation et l'entretien de la main-d'œuvre sont à la charge du secteur privé et d'ajouter : " Je ne fais aucun doute que le Roi d'Angleterre puisse construire 50 vaisseaux à la fois, sans embarrasser aucun des ports principaux dont toutes les ressources pourraient être dirigées vers les radoubs et les armements $»^{32}$. Autre information bien intéressante, Kersaint réfute l'idée, généralement répandue, selon lui, que I'Angleterre manque de bois. Tous les vaisseaux qu'il a vus sont construits en bois du pays et ils sont commandés très longtemps avant leur mise en chantier, les paiements se faisant à long terme. Les entrepreneurs construisent avec lenteur, " lorsqu'ils sont assurés dans un canton de la quantité de bois suffisante, ils contractent avec le gouvernement et l'ouvrage se commence $»^{33}$. Pour ces officiers, il est d'ailleurs indispensable de bien connaître la construction navale, les matériaux et les savoir-faire en usage dans les ports. Se former sur les chantiers en attendant un embarquement avait une visée pédagogique fondamentale car la maîtrise de I'outil naval chez ceux qui commandent un navire passe par cet apprentissage de la connaissance technique. C'est justement aux jeunes gardes de la Marine que s'adressent les différents manuels rédigés sur ces sujets désormais inclus dans leur programme de formation ${ }^{34}$. Rappelons également que les changements induits par I'application des nouvelles ordonnances sur l'organisation de la Marine, depuis Choiseul et surtout Sartine, rend

$31 \mathrm{lbid}$.

$32 \mathrm{lbid}$.

$33 \mathrm{lbid}$.

34 Citons à titre d'exemples les ouvrages de Duranti de Lironcourt, Instruction élémentaire et raisonnée sur la construction des vaisseaux, en forme de dictionnaire, Paris, Musier, 1771 ; François-Louis Du Maitz de Goimpy, Traité sur la construction des vaisseaux, Paris, Couturier, 1776 ; Charles Romme, L'art de la marine, ou principes et préceptes-généraux de l'art de construire, d'armer, de manœuvrer et de conduire des vaisseaux, La Rochelle, Chauvet, 1787 ; Honoré Vial du Clairbois, Traité élémentaire de la construction des vaisseaux, à l'usage des élèves de la Marine, composé \& publié, d'après les ordres de Monseigneur le Maréchal de Castries, Paris, Clousier, 1787. Voir Michel VergéFranceschi, Marine et éducation sous l'Ancien Régime, Paris, CNRS, 1991
I'outil naval aux militaires qui contrôlent désormais la direction des ports et des travaux de construction dans les arsenaux.Lors des voyages précédents, au XVIIe siècle, les rapporteurs présentaient un état des techniques en usages évaluées selon une méthode d'expertise, simple en apparence, qui distinguait les avantages des inconvénients. Dans la seconde moitié du XVIII siècle, c'est beaucoup plus le catalogage des nouveautés dans les procédés de fabrication et dans certaines opérations techniques qui devient déterminant. Les remarques du chevalier d'Oraison au sortir de la guerre de Sept Ans font apparaître des éléments de comparaison assez frappants s'agissant des magasins d'armement des vaisseaux, des ateliers, des fournitures navales et des installations portuaires. Les qualificatifs utilisés par d'Oraison renvoient à la propreté, la commodité, l'utilité et l'abondance. À Portsmouth, ce qu'il retient aussi est l'usage des pompes à chapelets pour vider les bassins de radoub et d'une pompe à moulin à vent servant à l'avitaillement en eau des navires :

«On y voit tous les magasins nécessaires pour serrer ce qui appartient à l'équipement des vaisseaux, mais sans luxe, ils ont toute l'utilité convenable, j'ai trouvé beaucoup de propreté dans l'intérieur au point que la corderie y est parquetée afin de ne point salir les cordes qui traînent en les travaillant, (...), on se sert pour achever de dessécher les bassins de pompes à chapelets qu'une roue tournée par des chevaux fait jouer, on voit de grands bassins pleins d'eau où sont les bois de mâture, c'est avec une machine flottante qu'on mâte vis à vis de l'arsenal (...) , l'on fait l'eau pour les vaisseaux à l'aide d'une pompe à moulin à vent (...), il est incroyable combien il y a de bois de tous les côtés pour la construction, de même pour les mâts et vergues en magasins $»^{35}$.

Le seul domaine dans lequel d'Oraison fait apparaître une supériorité française est celui de la conception des navires. II indique que les Anglais « qui n'avaient il y a dix ans aucun livre de tactique sur cette partie étudient aujourd'hui nos constructeurs et travaillent d'après nos modèles ». Le mot "chef d'œuvre » est même employé s'agissant des frégates françaises. Dans une lettre qui informe le ministre Choiseul de l'envoi de son mémoire, d'Oraison rassemble les informations les plus récentes sur ce qui constitue pour lui la vraie nouveauté, d'ordre stratégique, le doublage en cuivre des carènes :

"L'Amirauté n'a encore rien décidé à l'égard des plaques de cuivre dont on double aujourd'hui les vaisseaux, on a fait plusieurs différentes épreuves

35 AN, Marine B7 $475 n^{\circ} 11$ et 12, 1764-1765. 
plaçant tantôt les feuilles les plus épaisses de l'avant ou de l'arrière suivant que les uns ou les autres jugeaient que le frottement était plus considérable dans une partie que dans l'autre, le mastic est composé d'huile de baleine et de chaux $»^{36}$.

Il mentionne également dans sa lettre qu' « un machiniste a inventé une nouvelle pompe pour tirer l'eau de la cale » dont les amiraux ne veulent pas à bord des vaisseaux bien que son principal avantage soit « de diminuer la force nécessaire à son maniement tout en épuisant la même quantité d'eau que les pompes ordinaires ${ }^{37}$ ". C'est Rosily, qui demeurant deux années et demie en Angleterre, trouve et rencontre le mécanicien William Cole. Ce dernier fait même des propositions au ministre Sartine, Rosily ayant imaginé qu'il pourrait établir une manufacture en France. II a fourni des arguments solides pour la fabrication de ces pompes à chaînes qu'il juge bien supérieures aux «pompes royales » françaises. La rencontre avec le mécanicien anglais débouche sur une autre découverte, l'existence d'une autre machine « qu'il est défendu de montrer même aux Anglais » et pour laquelle William Cole a été employé. Cette machine qui « force les poulies en un clin d'œil, qui tourne et perce le fer avec plus d'aisance que nous tournons les bois en France » est propre "à mille autres choses pour la marine ${ }^{38}$. Walter Taylor, mécanicien de Southampton, a été aidé par William Cole pour construire sa machine. Voilà donc un autre mécanicien qu'il faudra rencontrer et persuader de travailler pour la marine du roi de France car les poulies de la fabrique de Taylor sont pour Rosily une des raisons de la supériorité des Anglais dans l'art de gréer leurs navires. Rosily indique à Sartine que Walter Taylor a dépensé 30000 livres pour construire sa fabrique et qu'il aurait gagné jusqu'à présent 400000 livres $^{39}$.

\section{6 lbid.}

37 Ces pompes sont décrites dans l'ouvrage de William Falconer, An universal dictionary of the marine, 1767 pour la 1 ère édition. Voir Brian Lavery, The ship of the line, vol. 2, Londres, Conway maritime press, 1983. II s'agit du perfectionnement de la pompe à chapelets, en usage depuis les années 1680 et de la pompe du mécanicien William Cole de 1761 (finalement adoptée par la Royal Navy). On comprend que les pompes n'intéressent pas seulement la marine, d'autres secteurs de l'industrie sont concernés par ces améliorations, ce qui explique que la Navy ait étudié de nombreux projets dans les années 1750, précise Brian Lavery.

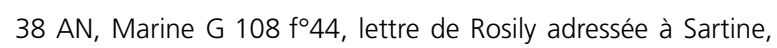
Londres, le 18 mai 1775.

39 Sylviane Llinares, "L'introduction de la mécanisation dans la fabrication des poulies au XVIII siècle », Chronique d'histoire maritime, $n^{\circ} 31,1995 / 1$, pp. 95-104. Sur la question des transferts de techniques et des inventions voir Liliane Hilaire-Perez,
Au sujet du gréement, il faut mentionner les remarques faites sur la qualité des cordages. D’Oraison était frappé par la propreté des corderies, Rosily décrit l'organisation du travail avec précision. II indique quels sont les appointements du commissaire et du maitre à la tête d'une corderie, quels sont les salaires des ouvriers fixes et de ceux « à marché » payés à la journée ou à la tâche. Les contrôles des chanvres et des goudrons, le soin apporté au filage et au goudronnage sont dépeints et présentés comme la raison majeure de l'excellence des cordages. Le maître doit veiller à ce que le « chanvre soit filé aussi fin et aussi égal qu'il est possible, que l'on fasse faire à la roue des révolutions égales et régulières, que les ouvriers fassent des pas égaux (...) $»^{40}$. La correspondance de Sartine avec Rosily révèle aussi l'existence d'un échange avec un « chimiste », le sieur Jackson, qui a mis au point un enduit pour durcir les bois. Rosily doit le convaincre de livrer « un ou deux pots de son enduit et des échantillons de ces bois durcis $»^{41}$.

Ce qui est vu dans les trois voyages est d'abord l'état des ports et le nombre de navires anglais. Les nouveautés sont vite repérées, le doublage en cuivre, les pompes et poulies, les peintures et enduits et enfin, ce que les officiers estiment tout aussi fondamental, les stocks de bois de construction et le recourt aux chantiers privés. La comparaison ne s'attarde pas sur l'architecture navale, dont on perçoit que la française a pleinement acquis son autonomie. Le comte de Kersaint, en 1785, après la guerre d'Amérique, accentue davantage l'idée de la perfection du navire anglais en espérant du « génie des inventions » de la France pour le concurrencer. ॥ traduit une première vision héritée d'un passé proche, et somme toute prévisible, qui confronte le bien construit anglais au bien conçu français, mais la comparaison va plus loin chez Kersaint pour mettre en avant l'excellence de l'adversaire. Le choix des mots et l'argumentation sont exemplaires : c'est d'abord l'imitation qu'il faut pratiquer en tous points, c'est le goût (l'intérêt) de la nation française pour sa marine qu'il faut développer, et enfin tout son talent créatif doit lui être appliqué. On comprend d'un point de vue historiographique ce qui a pu conforter la vision de la supériorité navale anglaise, comme s'il y avait en France, pays de terriens (?), une négligence permanente dans la finition et un détournement du génie industrieux au profit d'on ne sait quoi d'ailleurs sous la plume de Kersaint :

L'invention au siècle des Lumières, Paris, Albin Michel, 2000 et John R. Harris, Industrial espionage and technology transfer: Britain and France in the eighteenth century, Aldershot, Ashgate, 1998.

$40 \mathrm{AN}$, Marine G149 $\mathrm{n}^{\circ}$ 38, «Administration de la marine anglaise » décembre 1776 - mars 1777.

41 SHD Marine CC7 : dossier Personnel Rosily-Mesros. Lettre de Sartine, Versailles le $1^{\text {er }}$ septembre 1776. 


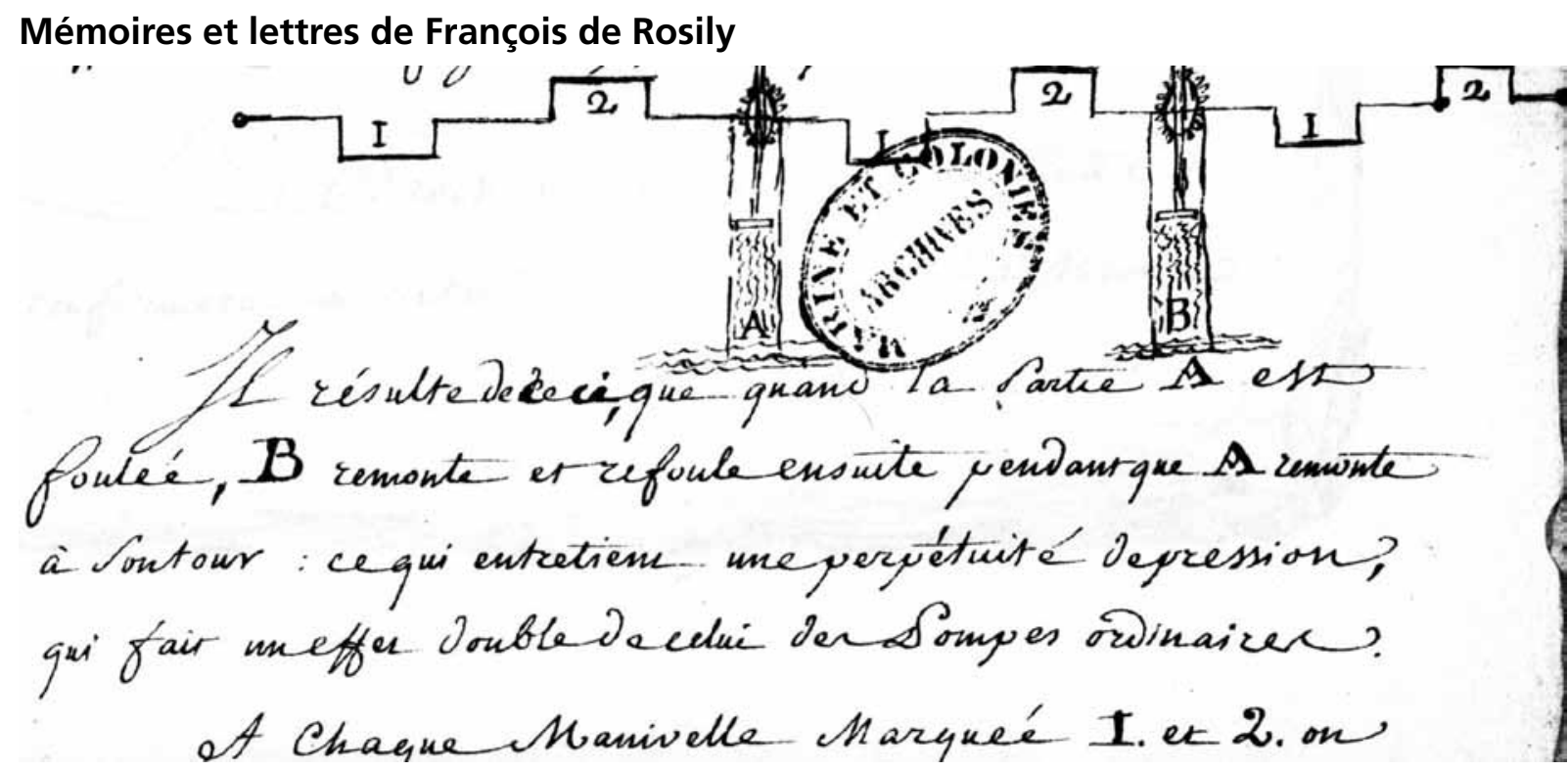

fig. 1 - AN Marine B7 475, n 16, vers 1775, Pompes à double manivelle, vues à Woolwich, lors de la visite du vaisseau l'Aigle de 64 canons

« Un vaisseau anglais de la construction actuelle en rade de Spithead a sous tous les rapports un modèle de perfection, le fini et la solidité, le soin et l'industrie qui ont donné à tout ce qui le compose des avantages (...), nous sommes sans doute de toutes les nations celle qui en approche le plus (...) Imitons leur exemple (...) occupons-nous sans relâche des moyens de leur enlever une supériorité qu'ils cherchent sans relâche à se procurer et si nous attirons les regards de la nation sur cet objet, espérons de son génie des inventions qui nous rendra à notre tour digne de leur servir d'exemple, en attendant ce moment qui serait certainement celui de notre plus grande gloire, voyons ce que I'Angleterre espère des nouvelles découvertes qu'elle a appliquées pour donner à son armée navale la supériorité la plus décidée sur celle de toute l'Europe confédérée $»^{42}$.

Kersaint pose ainsi deux questions majeures : comment parvenir au travail bien fait et comment obtenir les innovations ? Nul doute que toutes ces observations aient eu un certain écho auprès des dirigeants de la Marine dont l'objectif est de faire mieux, plus vite et moins cher depuis Colbert.

L'originalité de ces trois voyages faits par des officiers de la marine du roi est qu'ils n'ont guère d'équivalent, puisque ce sont les ingénieurs-constructeurs et les administrateurs, qui effectuaient depuis le XVIIe siècle

42 AN, Marine B7 $475 n^{\circ} 20$ et 21, « Vues générales sur l'état actuel de la marine d'Angleterre, août 1785 », introduction du mémoire. ces sortes de "Grand Tour » technicien en Hollande, en Angleterre et en Italie. Leurs correspondances et mémoires présentent la vision argumentée de la supériorité anglaise selon le domaine concerné (gréement, artillerie, matières premières, procédés de fabrication, manufactures, ate-liers, etc.). L'analyse du discours comparatif montre une évolution dans laquelle le bilan mesuré cède la place à la recherche de la nouveauté. II apparaît toutefois, vu de France, que la supériorité anglaise ne va pas de soi. Personne n'est dupe parmi nos observateurs, la maîtrise des mers est d'abord une question de moyens. Cette condition avérée de la puissance maritime n'interdit pas d'être curieux des progrès de son principal ennemi. La curiosité du perfectionnement technique exclut d'emblée I'œuvre de conception des navires dont la France veut s'enorgueillir. Par contre, elle stimule la recherche de tout ce qui peut contribuer à optimiser une marine, qu'il s'agisse de pompes pour les cales des navires et les bassins de radoub, des peintures, des poulies, etc. Ainsi, la curiosité française se focalise sur le mécanicien anglais, un personnage presque omnipotent tant la mécanique semble être le parangon de l'excellence technicienne. II reste que le catalogage de la technologie anglaise ne saurait à lui seul constituer la vision en creux de ce qui manque, de ce qui produit fatalement l'infériorité navale. L'observation met encore en relief les différences, mais elle débusque surtout les nouveautés, et c'est probablement là que se trouve le ressort du discours comparatif, une inquiétude face à la capacité d'innovation rapide, renou-velée et foisonnante de l'Angleterre. 


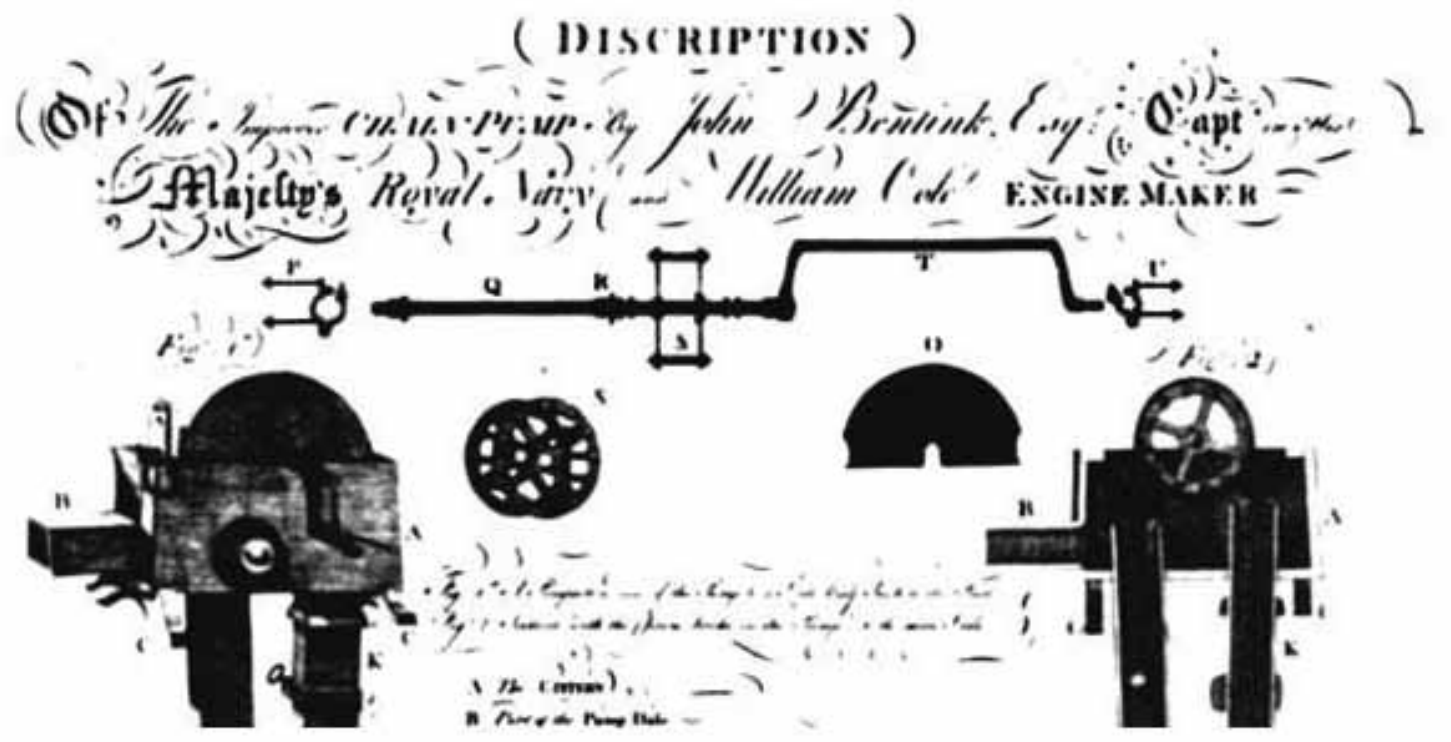

fig. 2 - AN Marine G 108 fº et 44, 18 mai 1775, « Plan d'une pompe de marine inventée par le Sr Cole » 